\title{
Two General Architectures for Intelligent Machine Performance Degradation Assessment
}

\author{
Yanwei $X u,{ }^{1,2}$ Aijun $X u,{ }^{1}$ and Tancheng $X i e^{1}$ \\ ${ }^{1}$ School of Mechatronics Engineering, Henan University of Science and Technology, Luoyang 471003, China \\ ${ }^{2}$ School of Mechanical Engineering, Tianjin University, Tianjin 300072, China \\ Correspondence should be addressed to Tancheng Xie; xietc@mail.haust.edu.cn
}

Received 5 June 2015; Revised 1 July 2015; Accepted 2 July 2015

Academic Editor: Dong Wang

Copyright (c) 2015 Yanwei Xu et al. This is an open access article distributed under the Creative Commons Attribution License, which permits unrestricted use, distribution, and reproduction in any medium, provided the original work is properly cited.

\begin{abstract}
Markov model is of good ability to infer random events whose likelihood depends on previous events. Based on this theory, hidden Markov model serves as an extension of Markov model to present an event from observations rather than states in Markov model. Moreover, due to successful applications in speech recognition, it attracts much attention in machine fault diagnosis. This paper presents two architectures for machine performance degradation assessment, which can be used to minimize machine downtime, reduce economic loss, and improve productivity. The major difference between the two architectures is whether historical data are available to build hidden Markov models. In case studies, bearing data as well as available historical data are used to demonstrate the effectiveness of the first architecture. Then, whole life gearbox data without historical data are employed to demonstrate the effectiveness of the second architecture. The results obtained from two case studies show that the presented architectures have good abilities for machine performance degradation assessment.
\end{abstract}

\section{Introduction}

Machine undergoes deterioration over time. In order to ensure its reliability, maintainability, and safety, timely condition monitoring and fault diagnosis are required. Temporal domain, frequency domain, and time-frequency domain based signal processing methods [1-3] are popular to detect fault related signatures for machine fault diagnosis. However, these methods need expertise. So, intelligent condition monitoring and fault diagnosis methods become attracting for identification of different machine health conditions [4].

Hidden Markov model (HMM) is a statistical model and it is successfully applied in speech recognition [5]. In machine condition monitoring and fault diagnosis, compared with Markov model, states used in HMM usually have no actual physic meaning and could not be directly observable. Nevertheless, state sequences in HMM can be estimated by a series of observation sequences, which may be features extracted from collected vibration signals. Bunks et al. [6] indicated that HMM is a very promising tool for constructing algorithms for condition based maintenance. Then, they investigated case studies related to Westland helicopter gearbox data set to demonstrate the potential benefit of HMM. Ocak and Loparo [7] proposed a HMM based fault diagnosis scheme by selecting a HMM with the highest probability. For bearing wear assessment, Ocak et al. [8] used a HMM to build a normal bearing model and observed any deviations from a normal bearing health condition for bearing performance degradation assessment. Ertunc et al. [9] applied a HMM to track tool wear conditions during drilling operations. Miao et al. [10] used empirical mode decomposition to analyze gearbox vibration signals. Then, a HMM was used for gearbox performance degradation assessment. Wang et al. [11] used two HMMs for early gear fault diagnosis and its performance degradation assessment, respectively.

In this paper, we present two architectures for machine performance degradation assessment by considering two different kinds of situations, with or without historical data. Machine performance degradation assessment aims to evaluate the deviation of the current health condition from the normal health condition and different fault levels. When historical data are available, they can be used to train 
different HMMs. Therefore, different health conditions and fault levels can be identified by finding the maximum log likelihood of the HMMs. Based on these HMMs, a slight fault can be initially identified. Then, based on this kind of fault pattern, different fault levels can be identified through multiple HMMs. On the other hand, in the case of unavailable historical data, an abnormal condition can only be inferred from any abnormal deviation of HMM from a normal condition. But it should be noted that the deviation can only be used to describe machine performance degradation trend rather than different performance degradation levels. Nevertheless, in some cases, some critical components, such as gears, may be directly replaced with new ones once an abnormal health condition is detected.

The rest of this paper is given as follows. Section 2 presents two architectures for machine performance degradation assessment under the situations with or without available historical data. In Section 3, two case studies are conducted to demonstrate the effectiveness of the presented architectures and to illustrate how they work. Conclusions are drawn at last.

\section{Two Architectures for Intelligent Machine Performance Degradation Assessment}

In this section, by considering two situations with or without historical data, two architectures are built by hidden Markov models for machine performance degradation assessment. One is an underlying state stochastic process, which can only be estimated by another observation stochastic process. A HMM can be expressed by a compact notation $\lambda=\{A, B, \pi\}$ and is introduced by the following [5]:

(a) $N$ is the number of hidden states in the hidden Markov model. Denote individual states by $S=$ $\left\{S_{1}, S_{2}, \ldots, S_{N}\right\}$ and the state at time $t$ by $q_{t}$.

(b) $M$ is the number of distinct observation symbols per state. The observation symbols correspond to the physical output of the system being modeled, such as features extracted from vibration signals. Denote the individual symbols by $V=\left\{V_{1}, V_{2}, \ldots, V_{N}\right\}$.

(c) The state transition probability distribution $A=\left\{a_{i j}\right\}$, where

$$
a_{i j}=P\left[q_{t+1}=S_{j} \mid q_{t}=S_{i}\right] \quad 1 \leq i, j \leq N .
$$

(d) The observation symbol probability distribution in state $j, B=\left\{b_{j}(k)\right\}$, where

$b_{j}(k)=P\left[v_{k} a t \cdot t \mid q_{t}=S_{j}\right] \quad 1 \leq j \leq N, 1 \leq k \leq M$.

If the observation is modeled as continuous, a continuous probability density function must be specified for each state. In general, a weighted sum of several Gaussian distributions is employed for continuous probability density function.

(e) The initial state distribution $\left\{\pi=\pi_{i}\right\}$, where

$$
\pi_{i}=P\left[q_{1}=S_{i}\right] \quad 1 \leq i \leq N .
$$

When HMM is applied, three problems should be solved. Firstly, how to evaluate a set of observations for a given hidden Markov model? This evaluation problem can be solved by Forward-Backward algorithm [5]. Secondly, how to find the optimal state sequence for a given set of observations? This decoding problem can be solved by Viterbi algorithm [5]. At last, how to find the optimal parameters of HMM for a given set of observations? The optimal parameters of HMM can be found by Baum-Welch algorithm [5].

2.1. An Architecture for Machine Performance Degradation Assessment with Historical Data. When there are historical data available, each machine health condition can be represented by a HMM. So, it is easy to distinguish different initial faults by selecting maximum log likelihood because HMM with maximum log likelihood indicates the current health condition of the machine. Here, taking different initial bearing faults, for example, bearings are prone to suffer from an inner race defect, an outer race defect, and a roller race defect. So, four HMMs are built to distinguish different bearing health conditions as shown in Figure 1(a). Once an abnormal health condition is detected, different fault levels can be estimated through Figure 1(b). In Figure 1(b), historical data with different fault levels are used to build HMMs. Therefore, HMM with maximum log likelihood can be used to indicate the current fault level. Machine performance degradation can be clearly assessed by the above procedure.

\subsection{An Architecture for Machine Performance Degradation} Assessment without Historical Data. When there are no fault historical data available, it is difficult to know which fault occurs firstly. But we can assess any log likelihood deviation from a normal condition as shown in Figure 2. In other words, only one HMM can be trained by normal data. Besides, only degradation trend rather than different fault levels can be assessed to judge how far the current health condition deviates from a normal condition. If a proper threshold is set, an abnormal condition can be detected and maintenance decision-making can be made. The proper threshold is usually set by expertise and users' requirement. The loop termination in Figures 1 and 2 is determined by users' requirements. It means that performance degradation levels are decided by users.

\section{Case Studies}

3.1. A Case Study with Historical Data. In this section, vibration data collected from a motor were used for our analyses. The sampling frequency was set to $12 \mathrm{kHz}$ [12]. Single point faults including a fault diameter of 0.007 inches, 0.014 inches, 0.021 inches, and 0.028 inches were, respectively, introduced to normal bearings using electrodischarge machining. For observation sequence extraction, discrete wavelet packet transform was employed to obtain wavelet packet node energy [8]. But here each wavelet packet node energy was scaled by a log algorithm for simplifying parameter estimation. Moreover, in order to enhance training sequence length, each original signal was divided into $L$ equal length 


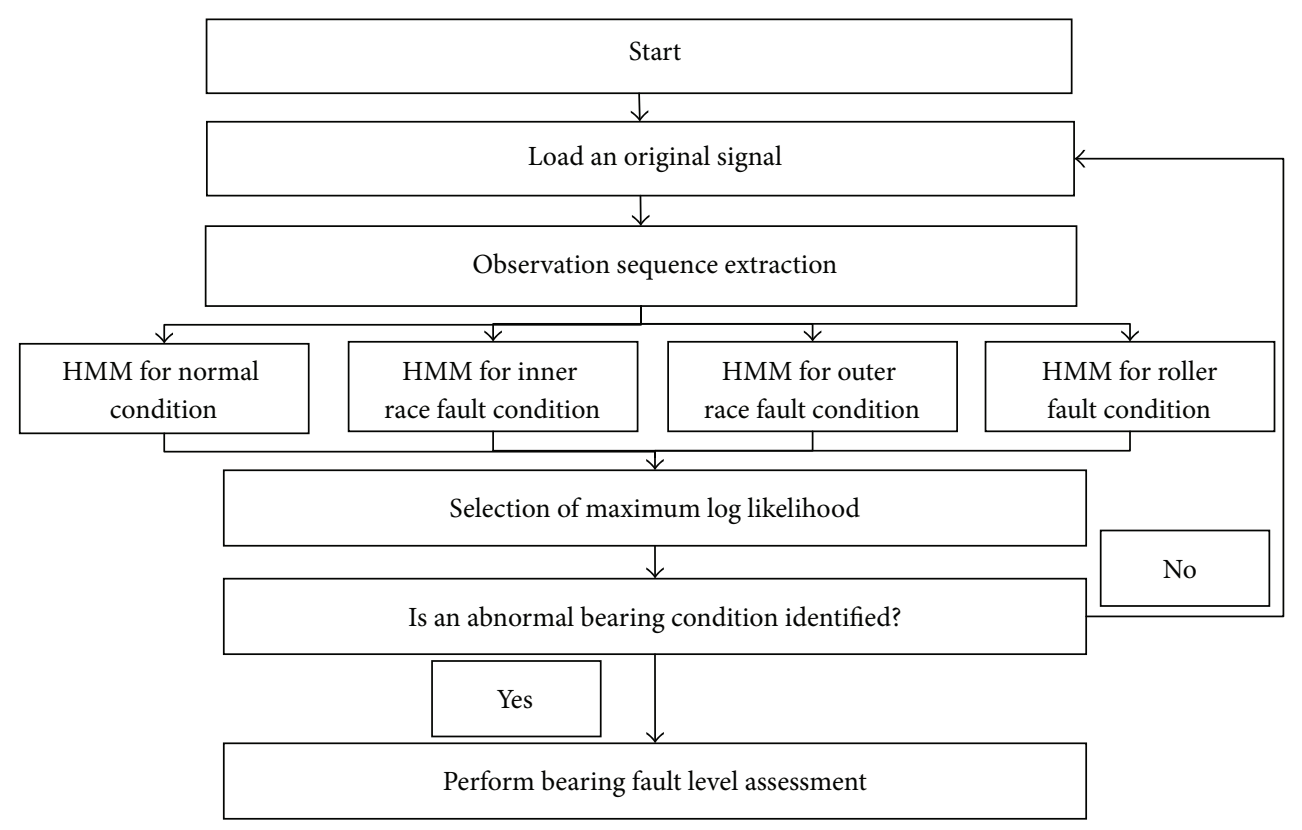

(a) Identification of an abnormal health condition

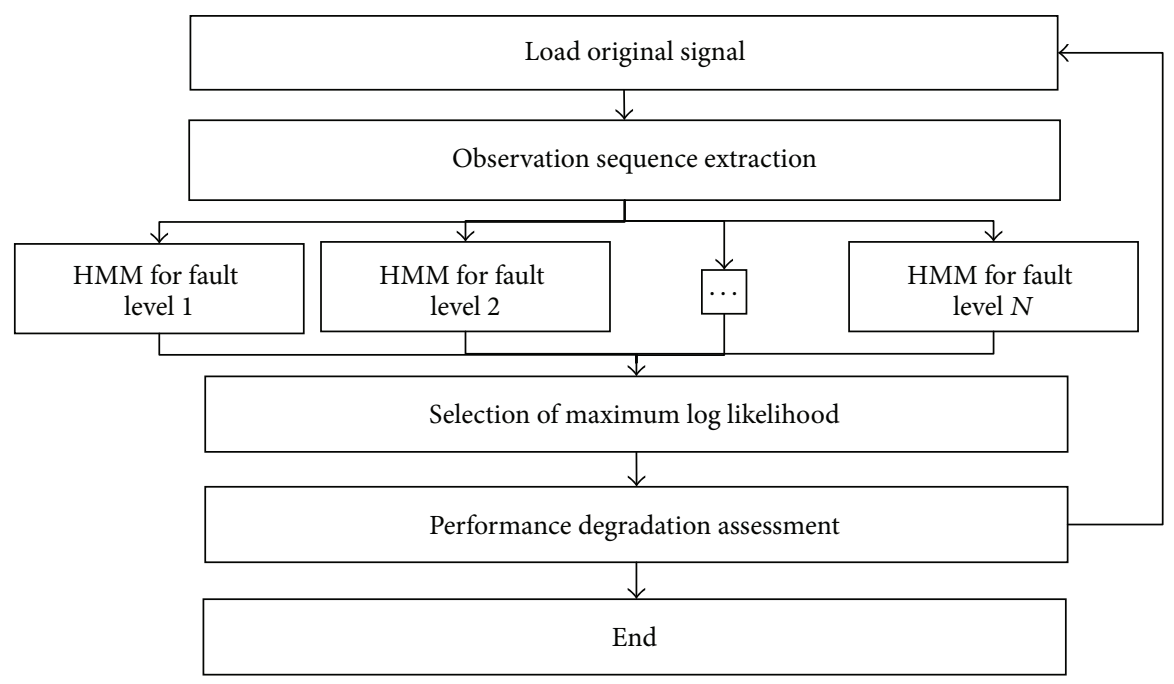

(b) Identification of different degradation levels

FIGURE 1: The presented flowchart for bearing performance degradation assessment in the case of available historical data.

subsignals before the use of discrete wavelet transform. In this paper, $L$ was set to 12 and bearing vibration signals with a length of 10 seconds were used. So, each subsignal contains 10000 samples. Daubechies-9 wavelet was chosen and wavelet packet decomposition level was 4 . The major reason for selection of Daubechies- 9 is that it has been proven to be effective in diagnosing localized bearing faults. In all, the size of the observation feature matrix is $12 \times 16$. Assume that hidden states are governed by a homogeneous Markov chain of order 1. HMM used here is a 5-state model with a diagonal covariance matrix containing 3 Gaussian mixtures. Then, firstly, in order to distinguish different bearing faults, four hidden Markov models are trained by normal bearing data, inner race fault data with a fault diameter of 0.007 inches, outer race fault data with a fault diameter of 0.007 inches, and roller race fault data with a fault diameter of 0.007 inches. Moreover, they are denoted by $\lambda_{1}, \lambda_{2}, \lambda_{3}$, and $\lambda_{4}$. Then, inner race fault data with a fault diameter of 0.007 inches is used for validation data. The diagnostic result is shown in Figure 3(a), where it is obvious that $\lambda_{2}$ has the maximum log likelihood among all HMMs $\lambda_{1}, \lambda_{2}, \lambda_{3}$, and $\lambda_{4}$. The result demonstrates that the HMMs are effective in identification of different initial defects.

Further, in order to assess bearing degradation, inner race fault with fault diameters of 0.007 inches, 0.014 inches, 0.021 inches, and 0.028 inches is used for further investigation. So, the size of the observation feature matrix is $48 \times 16$. Here, four HMMs are trained by inner race fault data with different fault 


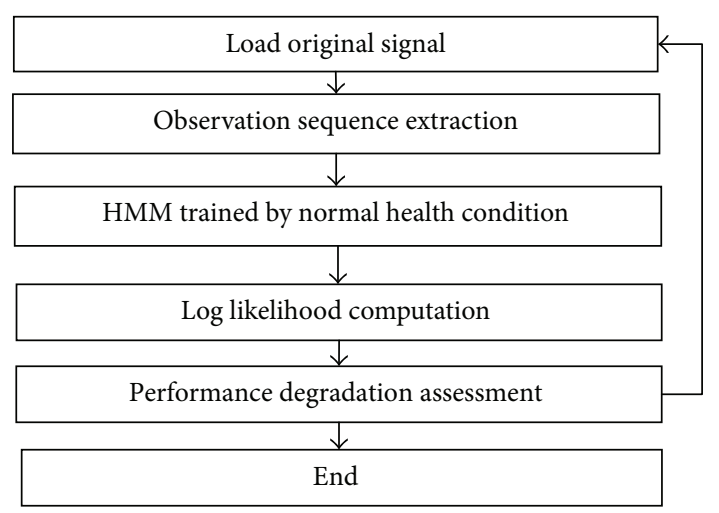

FIGURE 2: The presented flowchart for machine performance degradation assessment in the case of unavailable historical data.

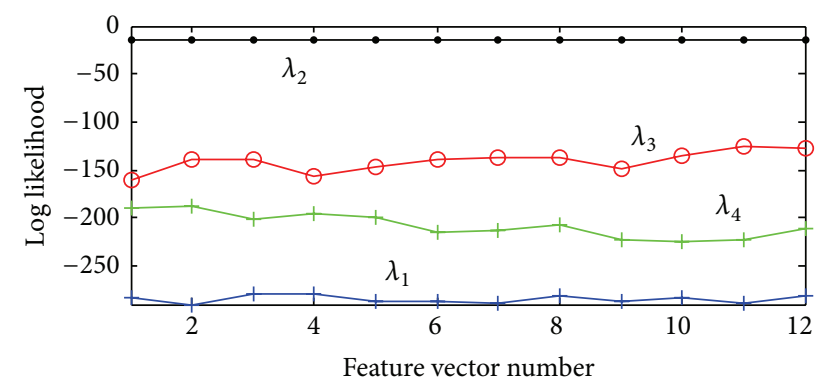

(a) Inner fault identification

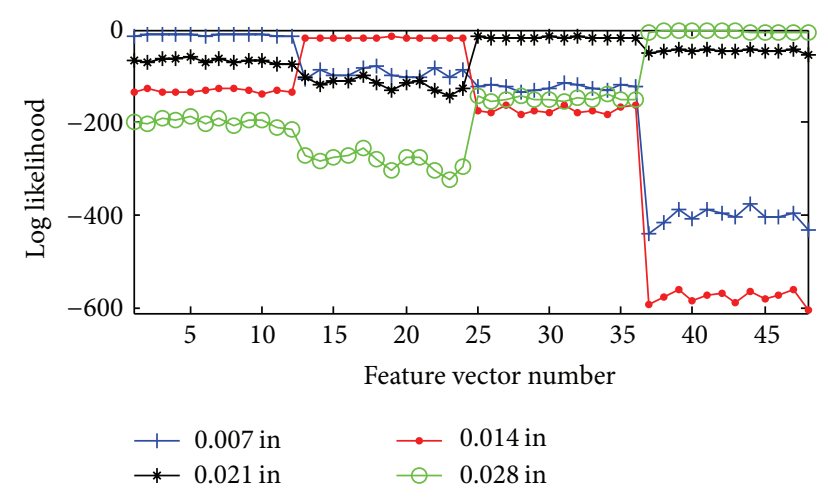

(b) Inner race different fault levels identification

FIGURE 3: Bearing performance degradation assessment using presented architecture 1 .

levels. The results are shown in Figure 3(b) using architecture 1. It is clear to see that the bearing with different inner fault levels can be well assessed by selecting the maximum log likelihood at different bearing inner race degradation stages.

3.2. A Case Study without Historical Data. In this section, a set of vibration signals collected from a run to failure gearbox were used to validate the second architecture in the case of unavailable historical data. The sampling rate was set to $20 \mathrm{kHz}$. The gear in a normal health condition ran to failure. A total of 148 data files were collected. Finally, the gear was subject to distributed faults. For observation sequence extraction, the same procedure was conducted as

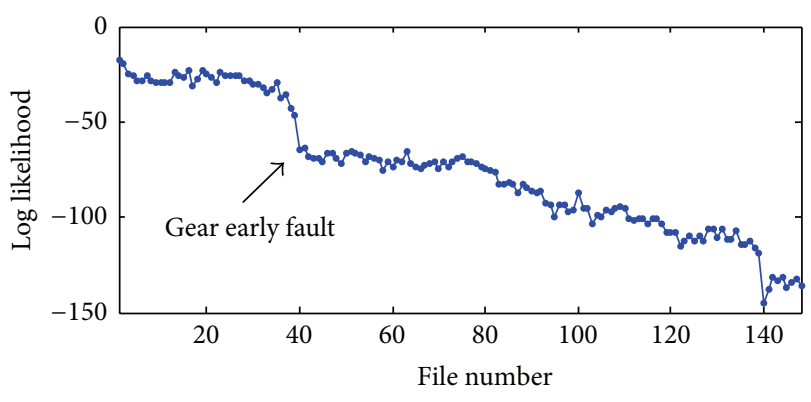

FIGURE 4: Gear performance degradation assessment using presented architecture 2 .

aforementioned step in Section 3.1. $L$ was set to 10 and gearbox vibration signals with a length of 10 seconds are used. So, each subsignal contains 20000 samples. Daubechies-9 wavelet was chosen and wavelet packet decomposition level was 4 . In all, the size of the observation feature matrix is $10 \times 16$ at each inspection. There is only one hidden Markov model trained by a normal condition. Then, the observation feature matrices at other inspection times serve as the input to the HMM trained by the normal health condition. The gear performance degradation trend can be observed by any deviations from the normal log likelihood. For slightly normal wear, the trend keeps decreasing. For an abnormal health condition, there is a sudden change indicated in Figure 4, which can be regarded as early gear faults because of its abnormality. Without historical data, only gear degradation trend can be observed and specific gear degradation levels are unknown.

\section{Conclusions}

In this paper, two architectures were presented for machine performance degradation assessment, by considering two kinds of situations, with or without historical data. For available historical data, different HMMs could be built to respond to different health conditions, including different faults and their associated fault levels. So, different initial fault patterns could be identified firstly. Then, their associated fault levels could be further assessed. On the other hand, for unavailable historical data, only normal data could be employed to construct one HMM that represents a normal condition. Any deviations from the normal condition could be observed. If a large sudden change occurs, it indicates the occurrence of the abnormal condition. However, it should be pointed out that the HMM without historical fault data can only be used to describe the performance degradation trend rather than specific performance degradation levels.

\section{Conflict of Interests}

The authors declare that there is no conflict of interests regarding the publication of this paper. 


\section{Acknowledgments}

The authors are grateful for the financial support of the National Natural Science Foundation of China (51305127) and the scientific research key project fund of the Education Department of Henan Province of China (14A460018). The authors would like to thank Professor K. A. Loparo for providing the bearing data.

\section{References}

[1] X. Zhang, J. Kang, L. Xiao, J. Zhao, and H. Teng, "A new improved Kurtogram and its application to bearing fault diagnosis," Shock and Vibration, vol. 2015, Article ID 385412, 22 pages, 2015.

[2] D. Wang, P. W. Tse, and K. L. Tsui, "An enhanced Kurtogram method for fault diagnosis of rolling element bearings," Mechanical Systems and Signal Processing, vol. 35, no. 1-2, pp. 176-199, 2013.

[3] D. Wang, P. W. Tse, and Y. L. Tse, "A morphogram with the optimal selection of parameters used in morphological analysis for enhancing the ability in bearing fault diagnosis," Measurement Science and Technology, vol. 23, no. 6, Article ID 065001, 2012.

[4] C. Shen, D. Wang, F. Kong, and P. W. Tse, "Fault diagnosis of rotating machinery based on the statistical parameters of wavelet packet paving and a generic support vector regressive classifier," Measurement, vol. 46, no. 4, pp. 1551-1564, 2013.

[5] L. R. Rabiner, "A tutorial on hidden Markov models and selected applications in speech recognition," Proceedings of the IEEE, vol. 77, no. 2, pp. 257-286, 1989.

[6] C. Bunks, D. McCarthy, and T. Al-Ani, "Condition-based maintenance of machines using hidden Markov models," Mechanical Systems and Signal Processing, vol. 14, no. 4, pp. 597-612, 2000.

[7] H. Ocak and K. A. Loparo, "HMM-based fault detection and diagnosis scheme for rolling element bearings," Journal of Vibration and Acoustics, vol. 127, no. 4, pp. 299-306, 2005.

[8] H. Ocak, K. A. Loparo, and F. M. Discenzo, "Online tracking of bearing wear using wavelet packet decomposition and probabilistic modeling: a method for bearing prognostics," Journal of Sound and Vibration, vol. 302, no. 4-5, pp. 951-961, 2007.

[9] H. M. Ertunc, K. A. Loparo, and H. Ocak, "Tool wear condition monitoring in drilling operations using hidden Markov models," International Journal of Machine Tools and Manufacture, vol. 41, no. 9, pp. 1363-1384, 2001.

[10] Q. Miao, D. Wang, and M. Pecht, "A probabilistic description scheme for rotating machinery health evaluation," Journal of Mechanical Science and Technology, vol. 24, no. 12, pp. 24212430, 2010.

[11] D. Wang, Q. Miao, Q. Zhou, and G. Zhou, "An intelligent prognostic system for gear performance degradation assessment and remaining useful life estimation," Transactions of The ASMEJournal of Vibration and Acoustics, vol. 137, no. 2, Article ID 021004, 2015.

[12] CWRU, Bearing Data Center, seeded fault test data, http:// csegroups.case.edu/bearingdatacenter/pages/download-datafile. 

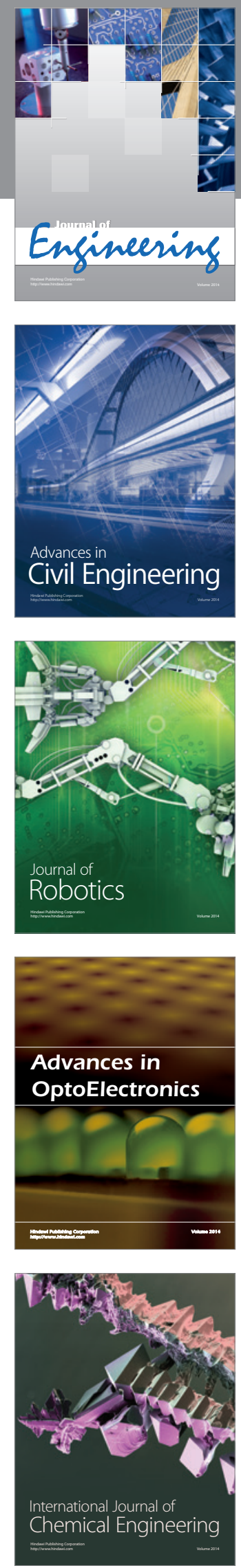

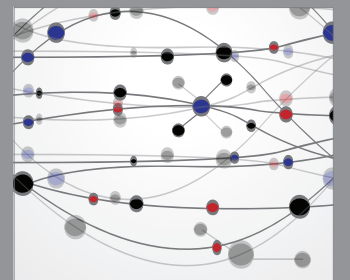

The Scientific World Journal
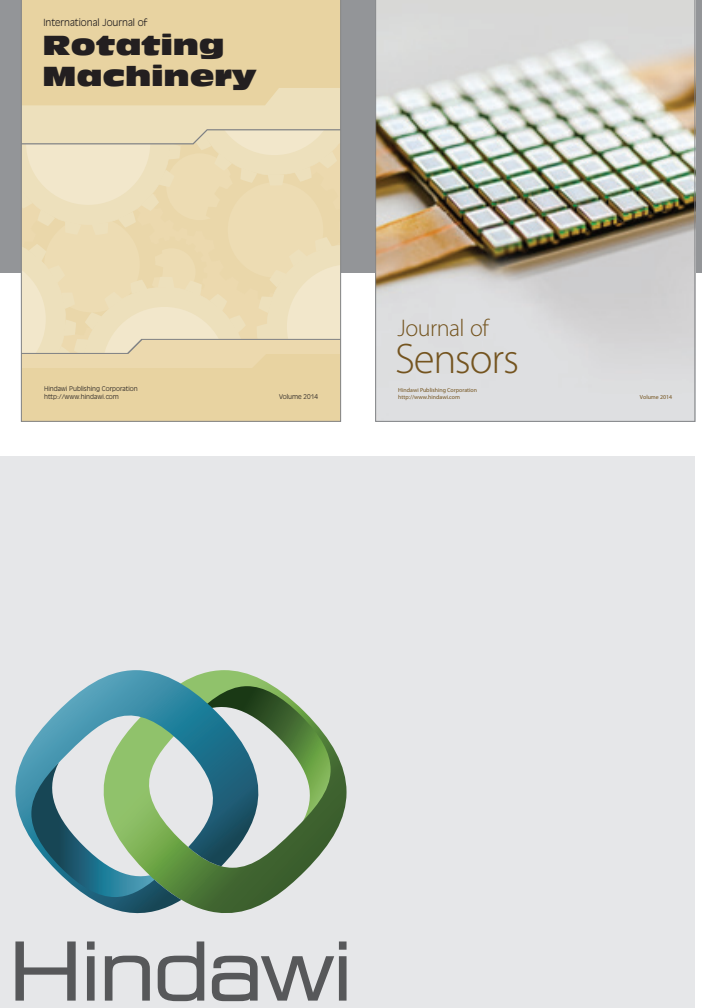

Submit your manuscripts at http://www.hindawi.com
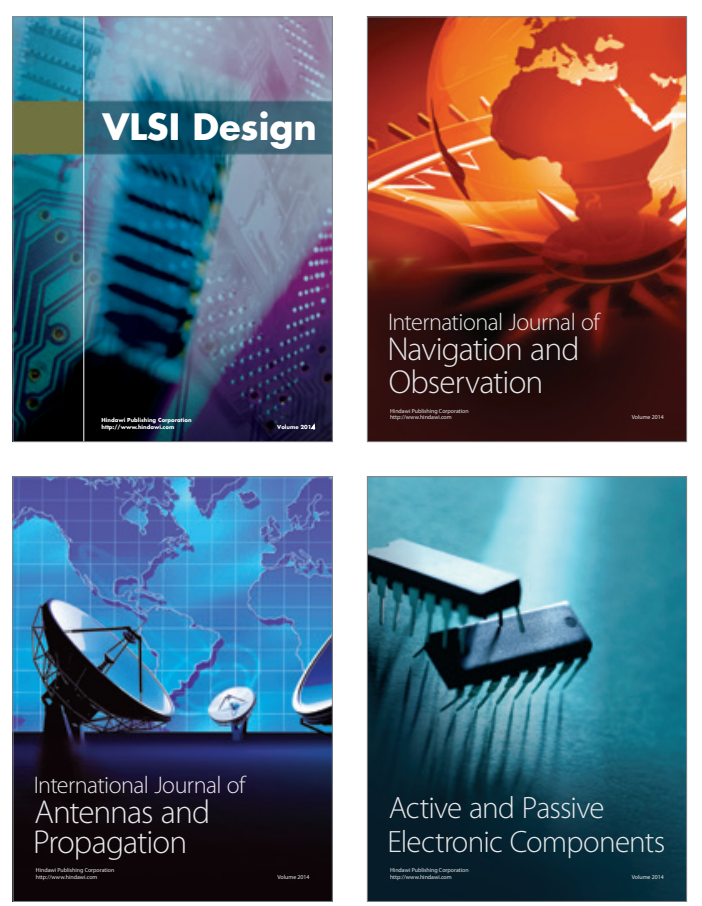
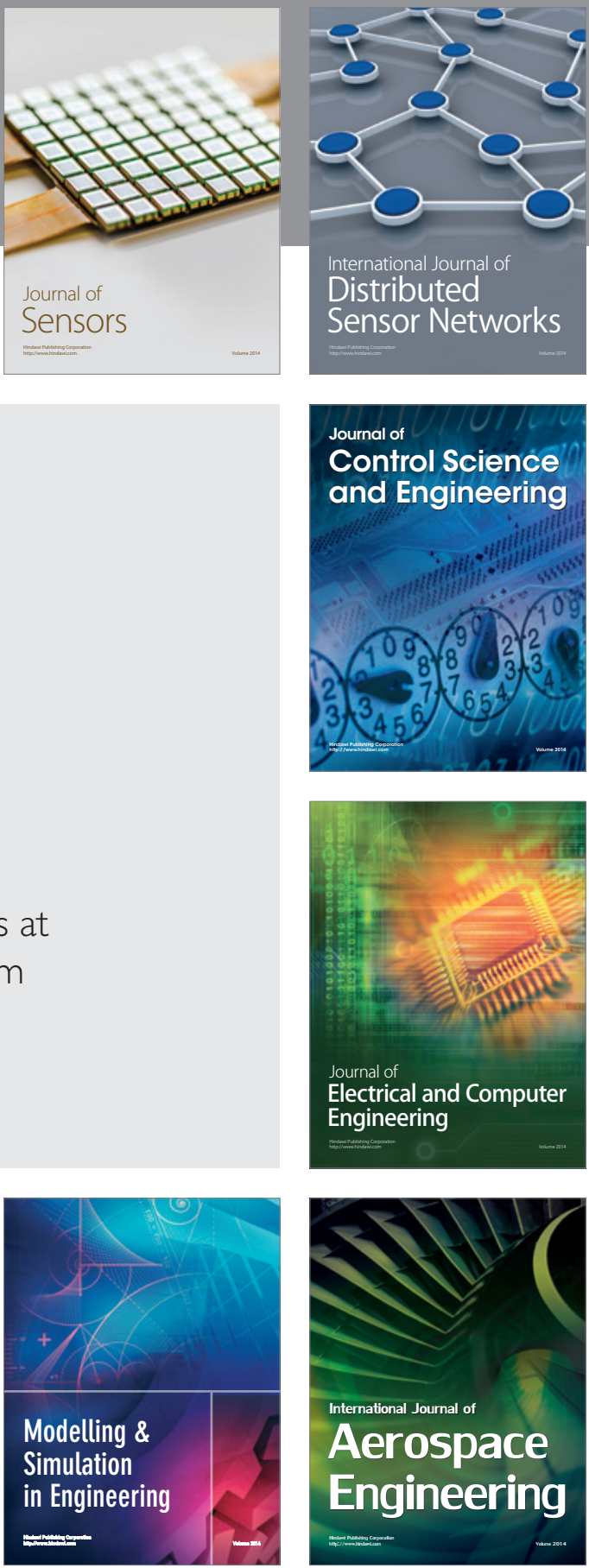

Journal of

Control Science

and Engineering
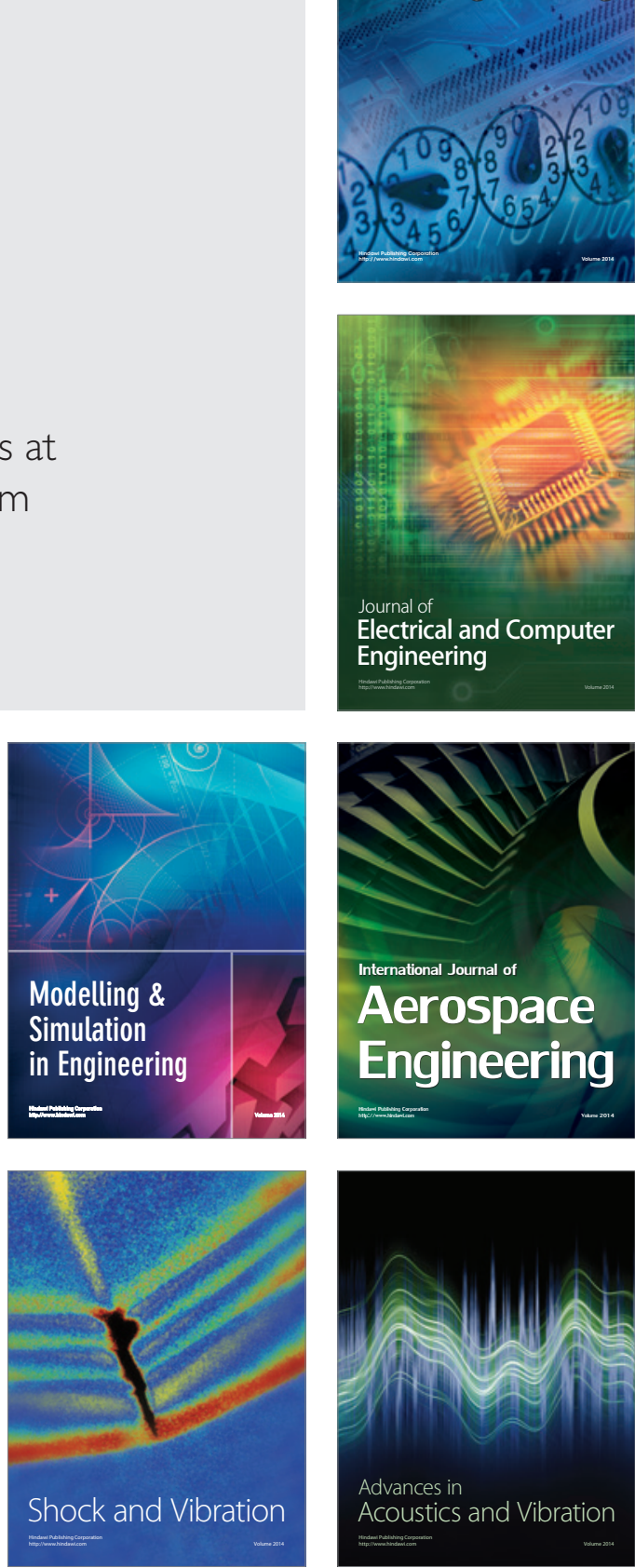